Scientific Instrument Research Association, under the auspices of which the work was conducted, under contract to that Department. Thanks are due to those physicists and mathematicians with whom I have enjoyed stimulating discussion, notable among whom are the late Dr. A. M. Turing, Mr. Brooker and Dr. Hoskin, of the University of Manchester ; Dr. H. H. Hopkins, of the Imperial College, London; and Mr. Bracey, of the above Association.

GORDON BLACK

Computing Machine Laboratory,

University, Manchester 13. Nov. 25.

\section{An Interpretation of the Long-Spacing Intensities of Calcium Stearate Monohydrate}

THE interpretation of powder patterns of soaps and other long-chain aliphatic compounds which cannot be obtained as single crystals is not easy and is usually ambiguous ${ }^{1}$. Samples of calcium stearate monohydrate prepared by D. Wallace using the method of Vold, Hattiangdi and Vold ${ }^{2}$ were recrystallized from solution in a mixture of propylene glycol and chloroform. The recrystallized soap was in the form of very tiny laths which could be formed into highly orientated sheets by sedimentation or by flotation. Such a specimen, mounted on a glass plate, used in a Geiger-counter diffractometer, gives rise to the long-spacing peaks of spacing $d=50 \cdot 30 \mathrm{~A}$. from planes parallel to the plane of the specimen and no other peaks.

The intensities of the reflexions were measured to order 30. No reflexions were found beyond this, although observations were continued beyond order 40. The higher-order reflexions were weak and digital counting was necessary for detecting and measuring them. The intensities, corrected for geometrical factors and sharpened, were used to calculate the linear Patterson function. This showed a prominent group of peaks centred at $17 \mathrm{~A}$. from the origin, probably indicating that in the structure there are two molecules separated by $17 \mathrm{~A}$. more or less parallel to their length. This model, assuming that the projection on the long-spacing axis is centrosymmetric, was used for the calculation of structure factors and the corresponding Fourier projection. The Fourier projection was refined using a semi-quantitative difference method ${ }^{3}$, and it was soon evident that the position of the water molecule, in projection, must be the same as that of the calcium atom. The final observed and calculated Fourier projections are shown in Fig. $1(a)$ and the corresponding suggested molecular arrangement in Fig. 1(b). Apart from the 'hole' at $z=\frac{1}{2}$ which is not present in the calculated Fourier projection, the agreement is good. The principal peaks and the trough at $z=0.32$ corresponding to the end of the molecule are satisfactorily reproduced in both projections.

The nature of the proposed structure with two molecules separated by $16 \cdot 8 \mathrm{~A}$. parallel to their length restricts ionic bonding between molecules to one direction only, unlike the two-dimensional ionic sheets observed in the soaps of monovalent metals.

The powder pattern can be indexed tentatively as a monoclinic unit cell using the methods of Vand, with $a=5.70 \mathrm{~A}$., $b=6.81 \mathrm{~A}$., $c=50.4 \mathrm{~A}$., $\beta=94^{\circ}$ and possible space group $P 2_{1}$. The observed and calculated densities are 1.07 and $1.04 \mathrm{gm} . \mathrm{cm}^{-3}$ respectively.
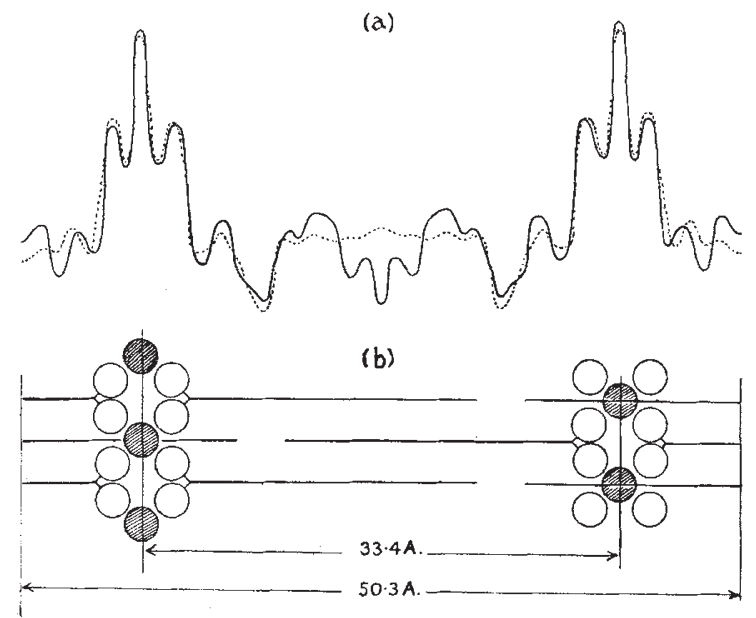

Fig. $1(a)$ Fourier projection on the long-spacing axis: the the brokcn line using the calculaied structure factors

(b) Possible type of structuro corresponding to Fig. $1(a)$. Calcium atoms, oxygen atoms and hydrocarbon chains are respectively. The water molecules have not been included in the diagram

The direction of the ionic bonding may be that of the $a$-axis, which is similar in length to the $b$-axis found by Vand, Lomer and Lang $^{5}$ in potassium laurate. The molecular cross-section of $19.8 \mathrm{A.}^{2}$ is greater than in potassium and silver soaps. This may be due partly to the restriction of the ionic bonding to one direction.

This work was supported by the U.S. Office of Ordnance Research under contract D.A. 04-495 ord303. I am grateful to Drs. M. J. Vold and R. D. Vold for the many stimulating discussions during the course of the work, which was done during the tenure of a Fulbright Travel Grant and a University of Southern California Fellowship.

Chemistry Department,

E. STANLEY*

University of Southern California,

Los Angeles 7. Aug. 30.

* Present address: Division of Physics. National Research Council, Ottawa, Canada.

1 Buerger, M. J., Proc. U.S. Nat. Acad. Sci., 31, 226 (1945).

"Vold, M. J., Hattiangdi, G. S., and Vold, R. D., J. Coll. Sci., 4, 93 (1949).

${ }^{3}$ Cochran, W., Acta Cryst., 4, 408 (1951).

- Vand, V., Acta Cryst., 1, 109 and 290 (1948)

${ }^{5}$ Vand, V., Lomer, J. R., and Lang, A., Acta Cryst., 2, 214 (1949).

\section{Formation of Compact Pieces of Grey Tin}

Is a recent communication on this subject, Groen ${ }^{1}$ described a method of preparing large pieces of grey tin by transforming an alloy of tin containing about 2 atomic per cent of mercury. Experiments have been proceeding in this Department with the same end in view, and the purpose of this communica. tion is to describe a possible explanation of this result.

In pure tin, the $\beta-\alpha$ transformation results in the formation of grey tin as a friable powder. This condition is the result of cracks, arising during the volume expansion associated with the formation of the $\alpha$-(grey) tin phase. Examination of partially transformed specimens has shown that the cracks may be classified into two groups. The first group appear between the islands of grey tin; the second 